\title{
Chemical and electronic properties of sulfur-passivated InAs surfaces
}

\author{
D.Y. Petrovykh ${ }^{\mathrm{a}, \mathrm{b}, *}$, M.J. Yang ${ }^{\mathrm{b}}$, L.J. Whitman ${ }^{\mathrm{b}}$ \\ a Department of Physics, University of Maryland, College Park, MD 20742, USA \\ ${ }^{\mathrm{b}}$ Naval Research Laboratory, Washington, DC 20375, USA \\ Received 3 July 2002; accepted for publication 7 October 2002
}

\begin{abstract}
Treatment with ammonium sulfide $\left(\left(\mathrm{NH}_{4}\right)_{2} \mathrm{~S}_{x}\right)$ solutions is used to produce model passivated $\operatorname{InAs}\left(\begin{array}{lll}0 & 0 & 1\end{array}\right)$ surfaces with well-defined chemical and electronic properties. The passivation effectively removes oxides and contaminants, with minimal surface etching, and creates a covalently bonded sulfur layer with good short-term stability in ambient air and a variety of aqueous solutions, as characterized by X-ray photoelectron spectroscopy, atomic force microscopy, and Hall measurements. The sulfur passivation also preserves the surface charge accumulation layer, increasing the associated downward band bending.
\end{abstract}

(C) 2002 Elsevier Science B.V. All rights reserved.

Keywords: X-ray photoelectron spectroscopy; Surface electrical transport (surface conductivity, surface recombination, etc.); Surface electronic phenomena (work function, surface potential, surface states, etc.); Etching; Oxidation; Indium arsenide; Sulphides

\section{Introduction}

A continuing challenge to the development of electronic and photonic devices based on III-V semiconductor heterostructures is the high density of electronic states within the band gap that typically exists at the surface of these materials. These mid-gap states pin the surface Fermi level $\left(E_{\mathrm{f}}\right)$ [1], creating a large Schottky barrier detrimental for metal contacts [2], and increasing the surface recombination velocity (a problem for photonic de-

\footnotetext{
${ }^{*}$ Corresponding author. Address: Code 6177, 4555 Overlook Avenue SW, Naval Research Laboratory, Washington, DC 20375-5342, USA. Tel.: +1-202-4043381; fax: +1-202-7673321.

E-mail address: dmitri.petrovykh@nrl.navy.mil (D.Y. Petrovykh).
}

vices). A widely studied approach to removing or at least controlling the $E_{\mathrm{f}}$ pinning is chemical passivation of surfaces $[1,3,4]$. Because GaAs has dominated III-V device development, most passivation research has focused on surfaces of this material $[3,5,6]$.

Instead of attempting to eliminate variations in mid-gap states on GaAs, it has recently been demonstrated that the sensitivity of these states to chemisorption can be used for gas sensing [7]. In a field-effect transistor (FET)-like device, the spacecharge layer in the GaAs creates a two-dimensional electron gas (2DEG) confined near the GaAs/AlGaAs interface about $50 \mathrm{~nm}$ below the GaAs surface. Adsorbates on the surface cause surface band bending and thus change the carrier density, which changes the conductance of the device. 
The inherent material properties of InAs suggest that at InAs surfaces a larger adsorbateinduced conductivity change can be observed. In InAs, $E_{\mathrm{f}}$ is typically pinned above the conduction band minimum (CBM), corresponding to downward band bending at the surface. This band bending causes a charge accumulation layer to naturally form at InAs surfaces and heterojunctions, as first indicated by Schottky barrier measurements $[2,8]$, and later confirmed by electron tunneling [9-11] and electron spectroscopy measurements [12-17]. The charge accumulation layer creates a natural 2DEG at InAs surfaces [9-11,16], and because the 2DEG is located immediately below the surface, surface band bending in InAs has been produced even by submonolayers of adsorbates [17-19].

In contrast with the above studies of InAs surfaces prepared under ultra-high vacuum (UHV) conditions, we are interested in sulfur-passivated InAs (InAs-S) surfaces prepared by wet chemistry. Wet chemical methods of surface passivation are inherently simpler than UHV-based approaches and offer many possibilities for subsequent surface processing, such as the addition of organic or biochemical functionality. In addition, as we demonstrate in this work, wet chemical sulfur passivation produces surfaces with a well-defined structure and robust chemical properties, appropriate for use as model InAs surfaces that can be easily prepared and studied in the ambient environment. In particular, we observe that the subsurface charge accumulation layer is preserved by the wet chemical treatment, and thus the passivated surfaces are suitable for measurements of adsorbate-induced band bending under the ambient conditions.

\section{Experimental}

Two types of InAs( $\left.\begin{array}{lll}0 & 0 & 1\end{array}\right)$ samples were used in this study. Surface chemistry and etching experiments were done with samples cut directly from a single-side polished undoped (intrinsically n-type) InAs( $\left.\begin{array}{lll}0 & 0 & 1\end{array}\right)$ wafer. InAs films grown by molecular beam epitaxy (MBE) were used for conductivity and electronic structure measurements, made possible by an InAs/AlSb quantum-well structure. The relaxed InAs films were grown on semi-insulating GaAs substrates in an MBE facility equipped with a valved As cracker and a Sb cracker. A buffer layer was first grown consisting of $1 \mu \mathrm{m}$ each of $\mathrm{GaSb}$ and $\mathrm{AlSb}$, followed by 10 periods of a (8 ML GaSb/8 ML AlSb) "smoothing" superlattice. Subsequently, $25 \mathrm{~nm}$ of AlSb was grown, followed by a $17 \mathrm{~nm}$-thick InAs terminal film. The sample was then cooled under As flux to $200{ }^{\circ} \mathrm{C}$. The growth surface was capped with $1 \mathrm{ML}$ of In deposited at $75^{\circ} \mathrm{C}$.

Commercial $20 \%$ water solution of analytical reagent grade ammonium sulfide $\left(\left(\mathrm{NH}_{4}\right)_{2} \mathrm{~S}\right)$ was used to prepare the sulfur-passivation solutions. Both the full strength $20 \%$ solution, $\mathrm{pH} \approx 11$, and that diluted by two parts of water, $\mathrm{pH} \approx 10-10.5$ (identified as " $7 \%$ solution" from here on) were used. Elemental sulfur was added to $\left(\mathrm{NH}_{4}\right)_{2} \mathrm{~S}$ to form sulfur saturated solutions, usually denoted as $\left(\mathrm{NH}_{4}\right)_{2} \mathrm{~S}_{x}$ in the literature [20]. Typically less than $0.1 \mathrm{~g}$ of sulfur dissolved in $15 \mathrm{ml}$ of the $7 \%$ solution, creating a bright yellow-colored liquid $(\mathrm{pH} \approx 9.5-$ 10). The InAs samples were de-greased in acetone and ethanol (or isopropanol) for $2 \mathrm{~min}$ each, rinsed in de-ionized water, and blown dry under dry nitrogen. In the standard passivation treatment, samples were soaked in $7 \%\left(\mathrm{NH}_{4}\right)_{2} \mathrm{~S}_{x}$ solution for 15 min at $\approx 35^{\circ} \mathrm{C}$. Passivating solution was heated in Pyrex glass beakers placed directly on a digital hotplate, or in polypropylene beakers placed in a waterbath. The samples were then rinsed for $2 \mathrm{~min}$ under flowing de-ionized water and blown dry.

The surfaces were characterized by X-ray photoelectron spectroscopy (XPS) with a monochromatized $\mathrm{Al} \mathrm{K} \alpha$ source in a commercial UHV system (base pressure of $1 \times 10^{-9}$ Torr). For highresolution core-level spectra, the analyzer pass energy was set to $20 \mathrm{eV}$; for survey scans, pass energies of 50 and $100 \mathrm{eV}$ were used. The corresponding nominal analyzer contributions to resolution were $0.36,0.9$ and $1.8 \mathrm{eV}$. The surface area sampled was about $0.5 \mathrm{~mm}^{2}$ for the standard measurement conditions and somewhat smaller for angle-resolved measurements $\left(5^{\circ}\right.$ nominal acceptance angle). All XPS measurements were done at room temperature with no additional in situ processing. 
To complement the angle-resolved XPS measurements of the surface electronic properties, the room-temperature, two-dimensional carrier density and zero-field mobility were determined by Hall measurements (in air) on $5 \times 5 \mathrm{~mm}^{2}$ samples from the MBE-grown InAs/AlSb material. The measurements were made using van der Pauw geometry and magnetic fields up to $3 \mathrm{kG}$.

\section{Results and discussion}

\subsection{Initial structure of S-passivated InAs}

An early review [21] of III-V semiconductorbased metal-insulator-semiconductor (MIS) structures makes the following comment about native oxides: "None of the homomorphic ... dielectric layers investigated thus far are entirely adequate for III-V compound MIS structures. The homomorphic layers are soft, hygroscopic, compositionally and structurally inhomogeneous." The native oxide on InAs thus cannot be considered as a good surface passivation layer and typically needs to be removed.

A common method of passivating III-V semiconductor surfaces is by immersion in sulfur-saturated ammonium sulfide solutions $\left(\left(\mathrm{NH}_{4}\right)_{2} \mathrm{~S}_{x}\right)$, as pioneered for GaAs [20,22,23], and later extended to other III-V materials $[1,4,24]$, including InAs [24-27]. As demonstrated by our XPS results (Fig. $1),\left(\mathrm{NH}_{4}\right)_{2} \mathrm{~S}_{x}$ passivation is very efficient at removing the oxides and preventing contamination for InAs $\left(\begin{array}{lll}0 & 0 & 1\end{array}\right)$. Surface contaminants after passivation, as indicated by intensity of the oxygen and carbon XPS peaks, are comparable (Fig. 1 insets) to those observed in a reference spectrum measured on an in situ cleaved surface [28]. Ratios of C $1 \mathrm{~s}$ and $\mathrm{O} 1 \mathrm{~s}$ intensities to the bulk-dominated As $3 \mathrm{~d}$ peak indicate that the passivated sample shows about $60 \%$ more oxygen but about $20 \%$ less carbon contamination than the reference sample; this result is quite remarkable given that the passivated sample was exposed to ambient air for about 20 min, whereas the reference sample was freshly cleaved in UHV [28].

Small amounts of physisorbed contaminants are, of course, unavoidable on surfaces prepared in

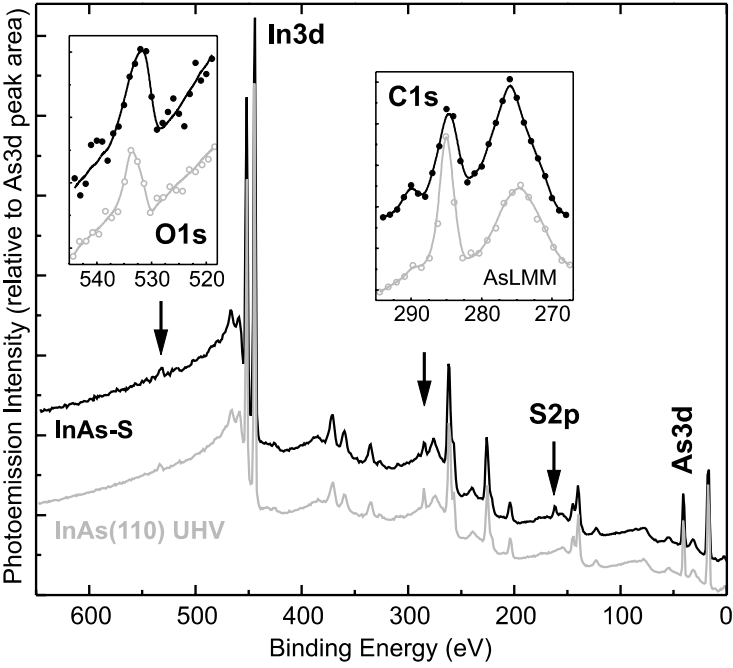

Fig. 1. XPS survey spectra for an $\operatorname{InAs}\left(\begin{array}{lll}1 & 1 & 0\end{array}\right)$ surface cleaved in situ (gray line, from Ref. [28]) and an $\left(\mathrm{NH}_{4}\right)_{2} \mathrm{~S}_{x}$-passivated In$\mathrm{As}\left(\begin{array}{lll}0 & 0 & 1\end{array}\right)$ wafer (black line). Except for the $\mathrm{S} 2 \mathrm{p}$ peak for InAs-S (arrow at $\approx 160 \mathrm{eV}$ ), the spectra are almost identical. The $\mathrm{O} 1 \mathrm{~s}$ and $\mathrm{C} 1 \mathrm{~s}$ regions are indicated by arrows $(\approx 530$ and $\approx 280 \mathrm{eV}$, respectively) and magnified in the insets. Even though the passivated sample spent $20 \mathrm{~min}$ in air, the oxygen and carbon contamination is comparable to that on the surface cleaved in situ.

the ambient environment, but because they are not likely to strongly affect the surface chemical and electronic properties, their presence does not preclude the use of InAs-S as a model passivated surface. Chemisorbed impurities (in particular oxides) are clearly efficiently removed by the passivation treatment, as indicated by the absence of In $3 \mathrm{~d}_{5 / 2}$ and As $3 \mathrm{~d}$ core-level components shifted to higher binding energy (compare Fig. 2 top and bottom).

The structure of the sulfur-passivated InAs ( $\left.\begin{array}{lll}0 & 0 & 1\end{array}\right)$ surface has been previously studied using spectroscopy [24,29] and diffraction [24,29,30] methods, resulting in the following model. Along the $\left(\begin{array}{lll}0 & 0 & 1\end{array}\right)$ direction, InAs is composed of alternating In and As planes. In XPS data (Ref. [29] and Fig. 2), In-S bonding is clearly observed, with little or no As-S. This chemical information suggests a surface terminated by an In plane with a disordered chemisorbed sulfur layer on top, consistent with coaxial impact collision ion scattering spectroscopy (CAICISS) data [30] and electron diffraction observations [24,29]. The observed 


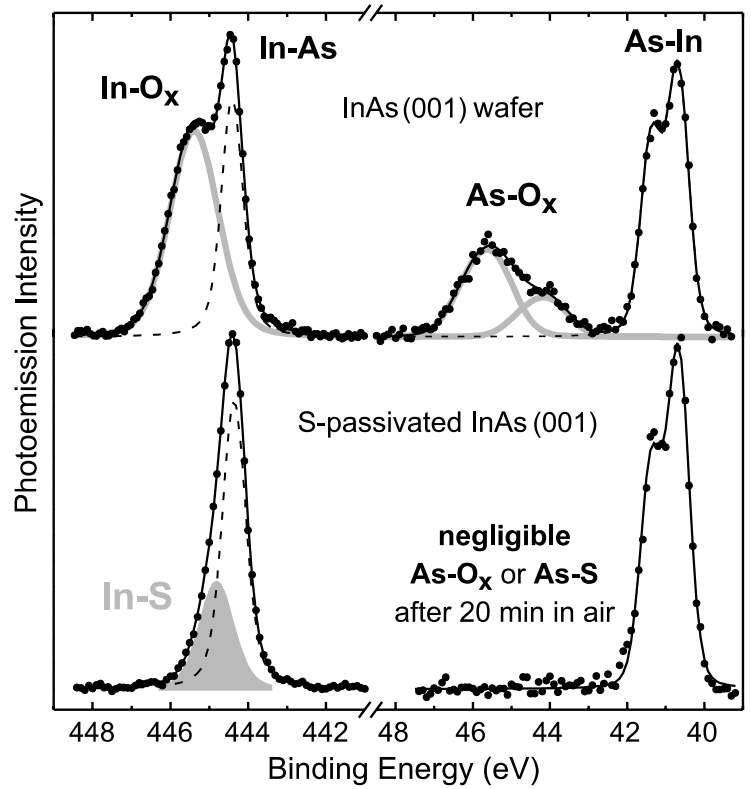

Fig. 2. Angle-resolved spectra (takeoff angle of $55^{\circ}$ ) of In $3 d_{5 / 2}$ and As $3 \mathrm{~d}$ core-levels for an $\operatorname{InAs}\left(\begin{array}{l}0 \\ 0\end{array}\right)$ wafer as-received (top) and after $\left(\mathrm{NH}_{4}\right)_{2} \mathrm{~S}_{x}$ passivation (bottom). Substantial native oxide $\mathrm{In}-\mathrm{O}_{x}$ and As- $\mathrm{O}_{x}$ components are present on the wafer as-received, shifted by 1 , and $3.2 \mathrm{eV}$ and $4.7 \mathrm{eV}$ from bulk peaks, respectively. After passivation (and $20 \mathrm{~min}$ in ambient air), In- $\mathrm{O}_{x}$ is replaced by an $\mathrm{In}-\mathrm{S}_{x}$ component (shifted $0.45 \mathrm{eV}$ ), and only negligible amounts of $\mathrm{As}-\mathrm{O}_{x}$ and As- $\mathrm{S}_{x}$ (intensity between 42 and $45 \mathrm{eV}$ ) are observed.

amount of sulfur is typically about one monolayer within the accuracy of the respective measurement techniques. There may also be a small amount of elemental As present in the top layer, but the first full As layer near the surface is protected by the In and $\mathrm{S}$ layers above it.

The subsurface position of the first full As layer and the corresponding essentially bulk-like As 3d XPS spectrum (Fig. 2) immediately after passivation are the two main features that, when coupled with robust and reliable preparation, make InAs-S a good model surface. Any chemisorbed adsorbates that are able to disrupt or bypass the top $\mathrm{S}$ and In layers can be detected by the appearance of chemically shifted components in As XPS spectra, thus providing a quantitative measure of the surface chemical stability (Section 4). Conversely, while the topmost As layer remains largely intact, the subsurface charge accumulation layer is likely to be preserved, as demonstrated in the next section.

\subsection{Electronic properties}

Although it is generally accepted that there is a charge accumulation layer at any InAs surface or interface prepared by any practical method under UHV conditions, the nature of the electronic states responsible for the $E_{\mathrm{f}}$ pinning above the CBM is a subject of ongoing debate $[18,19,31-33]$. It is thus unclear a priori whether the partially disordered chemisorbed layers added by the $\left(\mathrm{NH}_{4}\right)_{2} \mathrm{~S}_{x}$ passivation will quench, enhance or leave unchanged the degree of band bending. To determine the effect of this treatment we have used a combination of conductivity measurements, XPS, and self-consistent band bending calculations. Because of the small band gap in this system, accurate measurements of the band bending become very challenging. Therefore, we only summarize the results here; the methods and calculations will be described in more detail in a separate publication [34].

For a freshly passivated MBE-grown InAs(llll $\left.\begin{array}{lll}0 & 1\end{array}\right)$ film, the 2D carrier density determined by room-temperature Hall measurements was $3.3 \times$ $10^{12} \mathrm{~cm}^{-2}$, higher than the $1.9 \times 10^{12} \mathrm{~cm}^{-2}$ measured for the as-grown film. The carrier densities indicate a stronger band bending at the passivated than at the as-grown surface. Exposure to air during the measurements caused negligible oxidation of the InAs-S sample (e.g. see Fig. 2 bottom). The as-grown sample became slightly-oxidized, which was expected to increase the band bending compared to a clean InAs surface [17].

As a complement to the conductivity measurements, we also recorded high-resolution angleresolved XPS spectra of the As $3 \mathrm{~d}_{5 / 2}$ core-levels on these MBE films. The As $3 \mathrm{~d}_{5 / 2}$ binding energies, measured relative to the $\mathrm{Au}$ 4f peak at $84.00 \mathrm{eV}$, are shown in Fig. 3. The observed angular dependences indicate that some band bending is present in both cases. The surface sensitivity (i.e. the depth accounting for $95 \%$ of the signal) changes from about $10 \mathrm{~nm}$ for a $90^{\circ}$ photoelectron take-off angle (normal emission) to about $3 \mathrm{~nm}$ for a $25^{\circ}$ take-off angle. Even at glancing incidence then, the spatial resolution is still comparable to 


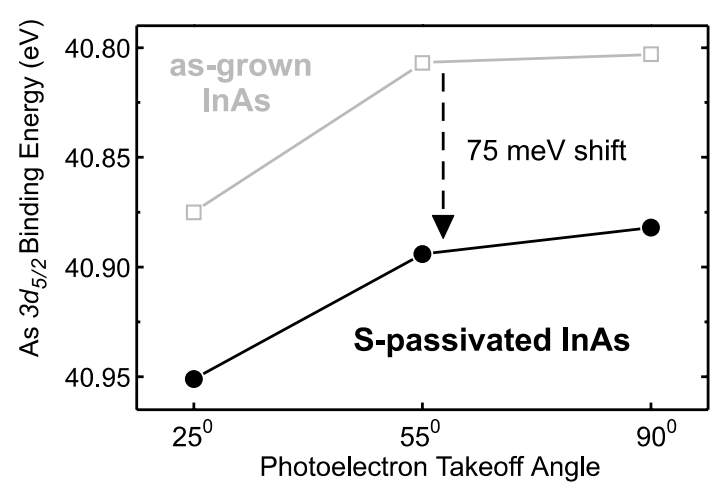

Fig. 3. As $3 d_{5 / 2}$ core-level binding energy vs. photoelectron takeoff angle for as-grown and $\left(\mathrm{NH}_{4}\right)_{2} \mathrm{~S}_{x}$-passivated InAs $\left(\begin{array}{lll}0 & 0 & 1\end{array}\right)$ films. The observation of an angular dependence indicates that some band bending is present in both cases, with the bands bent downward about $75 \mathrm{meV}$ more on the passivated surface.

the depth of the accumulation layer, so the apparent As $3 \mathrm{~d}_{5 / 2}$ peak position only represents some average band bending that is therefore smaller than the actual surface value. However, the consistent core-level shift to higher binding energy for the passivated vs. as-grown films, is an accurate measure of the relative band bending, which is about $75 \mathrm{meV}$ greater (downward) for the passivated film. A similar value, $60 \mathrm{meV}$, is obtained from self-consistent band bending calculations based on the Hall measurements data, which we consider to be good agreement given the experimental and theoretical uncertainties.

Both XPS and conductivity measurement thus indicate that the band bending is preserved after the passivation and that it is larger at passivated surfaces compared to a slightly oxidized one. Both techniques also indicate that the passivationinduced band bending is reduced by re-oxidation (in ambient or by oxygen plasma). This is a somewhat non-intuitive result given the higher electronegativity of $\mathrm{O}$ compared to $\mathrm{S}$. However it is consistent with a report [35] that Se-passivation, which is believed to be similar to S-passivation, induces "anomalously" large band bending as well.

\subsection{Re-oxidation in ambient environments}

Quantitative measurements of surface stability after a passivation treatment are most often done using characteristics (e.g. $I-V$ curves) of devices based on the passivated semiconductor; e.g., for InAs, metal-oxide-semiconductor structures [36] or photodetectors [37]. Another common method $[1,4]$ is to use the evolution of the photoluminescence yield to track the device stability over time [37]. Monitoring the oxidation of sulfur-passivated surfaces provides a more general device-independent method of characterizing the surface stability, e.g. oxidation in ambient air and by UV/ozone treatment has been studied for GaAs [38] and InP [39].

For InAs, XPS of elemental core levels is a sensitive method for characterizing re-oxidation, as indicated by the observation of shifted components in the In $3 \mathrm{~d}_{5 / 2}$ and As $3 \mathrm{~d}$ core-level spectra presented in Fig. 2. In particular, the As $3 \mathrm{~d}$ peaks for the As-oxide and sulfide exhibit substantial chemical shifts, 3-5 and $2 \mathrm{eV}$ [29], respectively, giving rise to well-separated peaks that can be accurately and reliably fit. The In $3 \mathrm{~d}_{5 / 2}$ sulfide- and oxide-related components are not as well separated from each other and the bulk peak $(0.45 \mathrm{eV}$ for In-S vs. $\approx 1 \mathrm{eV}$ for $\mathrm{In}-\mathrm{O}_{x}$ ), but In oxidation is associated with visible asymmetry of the In $3 \mathrm{~d}_{5 / 2}$ peak towards higher binding energy.

The re-oxidation of passivated surfaces in ambient air and de-ionized water, as revealed by the In and As 3d levels, is shown in Fig. 4. An almost identical increase in intensity on the higher binding energy side of the In $3 \mathrm{~d}_{5 / 2}$ peaks, compared to a freshly passivated surface, indicates essentially identical re-oxidation of the surface In under all ambient conditions. In contrast, the As $3 \mathrm{~d}$ peaks show a notable variation: whereas some As- $\mathrm{O}_{x}$ is present after three days of exposure to air, heating the sample in air for 15 min produces a significantly larger oxide peak, and the sample stored one day in water shows essentially no As oxidation.

To evaluate the longevity of the surface passivation effect, in Fig. 5 we compare the timedependence of the As- $\mathrm{O}_{x}$ intensity for samples stored in ambient air. It is clear that the S-passivation retards re-oxidation considerably. After a few days, the passivated samples show only about $1 / 3$ of the oxide level of the HCl-etched control. After over a month in the ambient air, the 


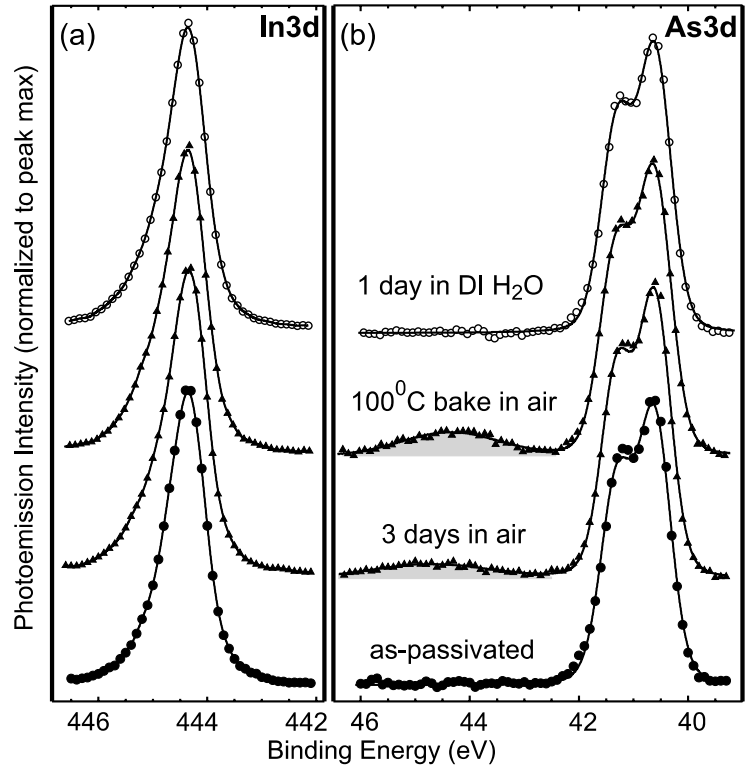

Fig. 4. The re-oxidation of $\left(\mathrm{NH}_{4}\right)_{2} \mathrm{~S}_{x}$-passivated $\operatorname{InAs}\left(\begin{array}{ll}0 & 0\end{array}\right)$ in air and water monitored via (a) In $3 \mathrm{~d}_{5 / 2}$ and (b) As $3 \mathrm{~d}$ corelevels (angle-integrated, normal emission). As- $\mathrm{O}_{x}$ re-growth is slow in ambient air at room temperature, but is considerably enhanced by a $15 \mathrm{~min}$ bake at $100{ }^{\circ} \mathrm{C}$. As- $\mathrm{O}_{x}$ formation in deionized water is negligible. The formation of $\mathrm{In}-\mathrm{O}_{x}$ is essentially identical in all three cases.

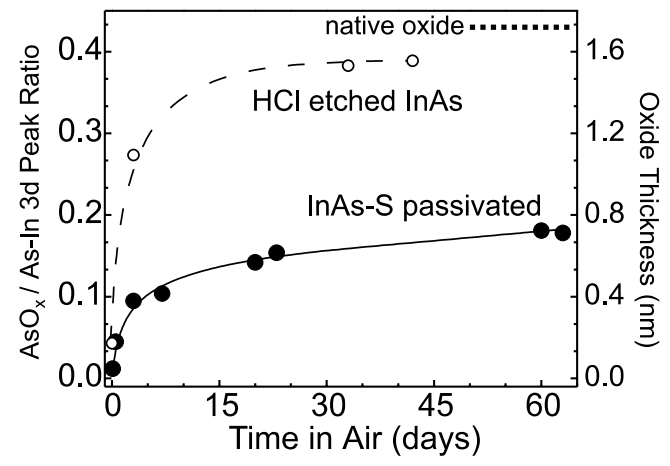

Fig. 5. Comparison of re-oxidation of the passivated (full circles) and HCl-etched (open circles) surfaces in ambient air, as indicated by the As- $\mathrm{O}_{x}$ peak intensity normalized to the bulk As peak (from angle-integrated, normal emission spectra). Lines are added to guide the eye. The normalized $\mathrm{As}-\mathrm{O}_{x}$ peak intensity scales approximately linearly with effective oxide thickness estimated from Ref. [40] data (right axis). The As- $\mathrm{O}_{x}$ amount for the $\mathrm{HCl}$-etched sample almost reaches that of an $\operatorname{InAs}\left(\begin{array}{lll}0 & 0 & 1\end{array}\right)$ wafer as-received (dotted line).

passivated samples develop less than half of the native oxide thickness (Fig. 5 dotted line), whereas the acid-etched control is almost fully re-oxidized. It should be noted that the initial amount of As- $\mathrm{O}_{x}$ observed immediately after the $\mathrm{HCl}$ etch is comparable to the passivated sample only because the surface is covered with an amorphous layer of elemental As and other by-products of the acidetch reaction.

The XPS intensity of the As- $\mathrm{O}_{x}$ component can be quantitatively converted into an effective layer thickness using a simple overlayer model and reference oxide thickness data from Ref. [40]. As shown on the right axis in Fig. 5, the oxide regrown on the passivated samples is very thin, $<1$ $\mathrm{nm}$. However, this thickness is extrapolated from data for oxides $>1 \mathrm{~nm}[40]$, which are likely to be more uniform and of somewhat different composition, so this calibration is only approximate. The native oxide layer appears to be about $1.7 \mathrm{~nm}$, a thickness within the range of the reference dataset [40] and therefore more reliable. For comparison, a previous spectroellipsometry study [41] estimated the thickness of the amorphous overlayer (including sulfur, water and oxides) to be about $3 \mathrm{~nm}$ on a S-passivated sample after $16 \mathrm{~h}$ in air.

\subsection{Chemical stability}

The spectra in Fig. 6 illustrate the reactivity of S-passivated surfaces in aqueous solutions of varying $\mathrm{pH}$. It is clear that changes from neutral $\mathrm{pH}$ to both higher and lower values produce changes in surface composition, i.e. the surface is not chemically inert. At higher $\mathrm{pH}$, In shows additional oxidation compared to that observed in water, whereas As is essentially unchanged. Lower $\mathrm{pH}$, on the other hand, essentially removes the oxide and sulfide components from In $3 \mathrm{~d}_{5 / 2}$ spectra, and introduces an As $3 \mathrm{~d}$ component centered around $42.5 \mathrm{eV}$ binding energy (highlighted in gray), which is consistent with an As-S chemical shift [29]. The observed behavior is consistent with the solubility properties of In and As compounds [42,43]: $\mathrm{AsO}_{x}$ and $\mathrm{AsS}_{x}$ are more soluble at high $\mathrm{pH}$ (similar to the $\mathrm{pH}$ of the passivating solution), $\mathrm{InS}_{x}, \mathrm{AsO}_{x}$, and $\mathrm{InO}_{x}$ are soluble at low $\mathrm{pH}$ (e.g. an acid bath strips the native oxide layer).

The stability of S-passivated surfaces was tested in the following commonly used organic solvents: 


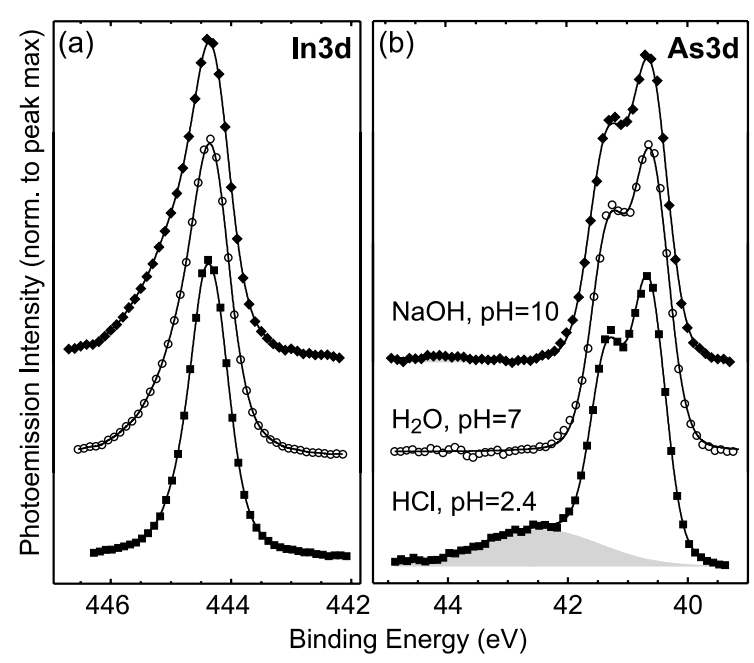

Fig. 6. Angle-integrated, normal emission In $3 \mathrm{~d}_{5 / 2}$ and $\mathrm{As} 3 \mathrm{~d}$ core-level spectra following the reaction of S-passivated InAs surfaces in aqueous solutions with a range of $\mathrm{pH}$. In de-ionized water, As- $\mathrm{O}_{x}$ formation is negligible and In oxidation is comparable to that in air (see Fig. 4). A dilute $\mathrm{HCl}$ solution removes In- $\mathrm{O}_{x}$ and $\mathrm{In}-\mathrm{S}_{x}$ almost completely, with the sulfur apparently transferred to the As (42-43 eV component). Dilute $\mathrm{NaOH}$ produces the strongest In oxidation along with a very slight increase in As- $\mathrm{O}_{x}$ (predominantly an $\mathrm{As}_{2} \mathrm{O}_{3}$ component at $44 \mathrm{eV}$ ).

ethanol, isopropanol, acetone, hexanes, toluene, ethyl acetate, tetrahydrofuran, methylene chloride, and chloroform (data not shown). The selected solvents span a range of values of the dielectric constant, a solvent property reported $[3,44,45]$ to affect passivation by sulfides (from 1.9 for hexanes to 25 for ethanol).

We find that the sulfur layer is not dissolved by any of the investigated solvents. Overnight soaking in toluene and hexanes, however, induced substantial oxidation of the sulfur, and produced the highest oxidation both in terms of $\mathrm{AsO}_{x}$ formation and total $\mathrm{O} 1 \mathrm{~s}$ intensity. In general, there is a trend of increasing oxidation with decreasing dielectric constant of the solvent, which most likely is related to oxygen diffusion and solubility. The carbon contamination was the highest after the soak in hexanes, about twice as much as after any of the other solvents. This contamination resisted rinsing and soaking in ethanol and methylene chloride, indicating the formation of a strongly adsorbed hydrocarbon layer.
All solvents were ACS and HPLC grade, used without any additional purification, the results may be different with highly purified solvents or under anaerobic conditions. We found that a small concentration of impurities in nominally benign solvents can strongly affect their reaction with a passivated surface; e.g., methylene chloride from a bottle open for over a year created more In and As oxides than in the native surface oxide, and almost completely removed the sulfur. The same solvent from a freshly opened bottle produced the smallest degree of oxidation in this study! Aged vs. fresh chloroform behaved in a similar way, and the aging effect could be replicated to some degree in fresh solvents degraded by exposure to UV light.

\subsection{Structure and reactivity}

Both the surface oxidation and chemical reactivity results support the standard S-on-In-on-As "layer-cake" structure model. A small and fairly constant degree of surface In oxidation is observed following various exposures to air and water (Fig. 4(a)), consistent with a partially exposed top In layer protected only by a disordered S layer. In contrast, the degree of As oxidation depends quite dramatically on the conditions (see Figs. 4(b) and 6(b)). In non-reactive liquid environments, As oxidation is slow and its rate increases with oxygen diffusion and solubility. In air, the As oxidation is faster, and the rate dramatically increases with temperature. Oxidation in both types of environment is consistent with the "layer-cake" structure model, whereby the As layer reactivity is limited by oxygen diffusion through the S-In overlayer, and thus As oxidation is faster at higher gaseous oxygen concentration and temperature.

\subsection{Implications for sensor applications}

There are three general approaches to improving sensitivity of a sensor device based on conductivity changes due to adsorbate-induced band bending (e.g. the GaAs/AlGaAs structure [7] described in the introduction): reducing the distance between the sensing layer and adsorbates (surface), having a $2 \mathrm{DEG}$ in the sensing layer, and reducing 


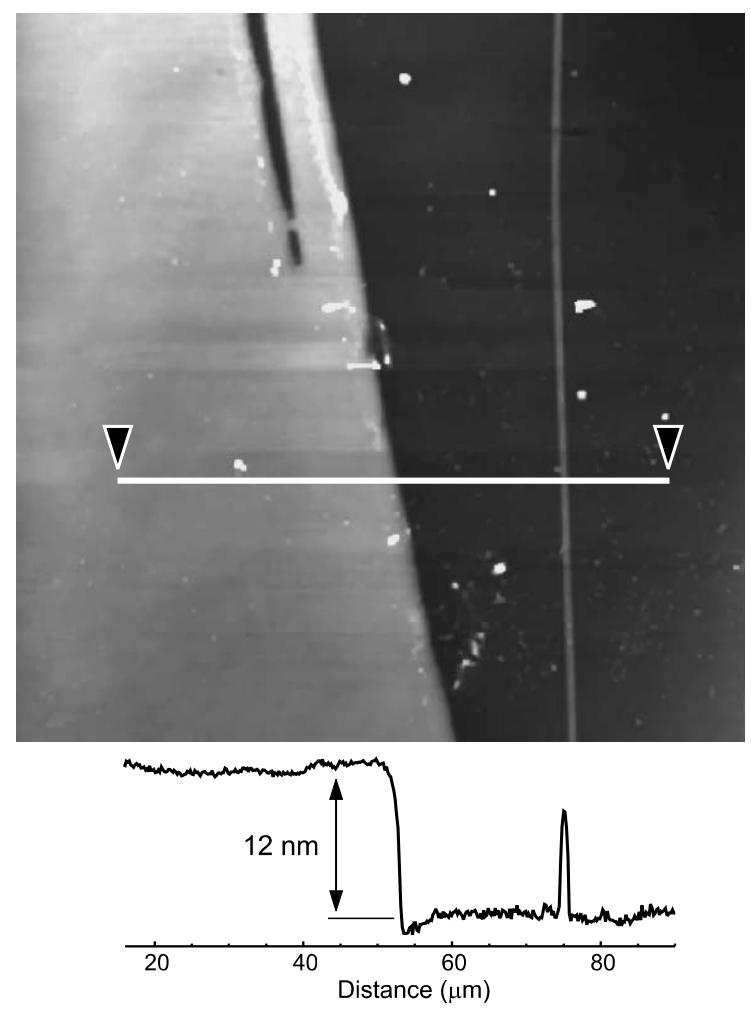

Fig. 7. Tapping mode AFM image and line scan profile across a $12 \mathrm{~nm}$ etch step on a $\left(\mathrm{NH}_{4}\right)_{2} \mathrm{~S}_{x}$-passivated InAs $\left(\begin{array}{lll}0 & 0 & 1\end{array}\right)$ surface. The upper terrace (left) was protected during passivation by PMMA resist which was removed prior to imaging. When applying the resist, a single stray brush hair produced the narrow line seen on the lower terrace (right), demonstrating process compatibility with micron-scale features.

the size of the sensing elements [46] to increase the surface-to-volume ratio.

The subsurface charge accumulation layer and the associated 2DEG are inherent properties of InAs surfaces. The $\left(\mathrm{NH}_{4}\right)_{2} \mathrm{~S}_{x}$ passivation treatment can be potentially extended for use on nanostructures such as InAs nanowires [47], because the InAs etch rate in $\left(\mathrm{NH}_{4}\right)_{2} \mathrm{~S}_{x}$ solution is only $0.8 \mathrm{~nm} /$ $\min (50 \mathrm{~nm} / \mathrm{hr}$ reported in [24]), as demonstrated in Fig. 7 by a $12 \mathrm{~nm}$ etch step after $15 \mathrm{~min}$ passivation. ${ }^{1}$ In addition, the large carrier density, on

\footnotetext{
${ }^{1}$ We estimate at least a 1:5 ratio between InAs and its native oxide etch rate. Between InAs and the two lattice matched materials $\mathrm{GaSb}$ and $\mathrm{AlSb}$ respective ratios are about 1:20 and $1: 50$.
}

the order of $10^{12} \mathrm{~cm}^{-2}[13,15]$, makes InAs surfaces inherently conductive even for nanometer-scale films [48-50] and wires [51,52]. InAs-based conductivity sensors therefore can potentially benefit from all the factors that would increase their sensitivity.

The $\left(\mathrm{NH}_{4}\right)_{2} \mathrm{~S}_{x}$ passivation effectively removes undesirable oxides and impurities with minimal etching, creates InAs-S surfaces with good shortterm stability in the ambient environment, and preserves the beneficial electronic surface properties - all requirements for a first step in a practical sensor surface preparation. Self-assembled monolayers (SAMs) offer a promising extension of this passivation strategy that may provide longer term stability $[53,54]$ and biocompatibility. Because the interface chemistry is similar to InAs-S, we can expect SAM passivation to preserve InAs surface electronic properties [55]. Possible strategies for SAM formation include the use of modified solvent-alkanethiol combinations for passivation [56], direct attachment of alkane chains to the InAs-S surface, or sulfur displacement by alkanethiols.

\section{Conclusions}

We have established that ammonium sulfide $\left(\left(\mathrm{NH}_{4}\right)_{2} \mathrm{~S}_{x}\right)$ treatment of InAs( 001$)$ surfaces produces a model passivated surface with well-defined structure and properties. The treatment effectively removes oxides and contaminants with minimal surface etching, and produces a covalently bonded sulfur passivation layer with good short-term stability in a variety of ambient environments. The sulfur passivation also preserves the surface charge accumulation layer, increasing the associated downward band bending. Given that $\left(\mathrm{NH}_{4}\right)_{2} \mathrm{~S}_{x}$ solutions are widely used for III-V semiconductor surface passivation $[1,4]$, the systematic evaluation of the surface stability and chemical properties of the model surface in this work establishes a good practical benchmark for evaluating alternative passivation approaches. The electronic properties of the model S-passivated surfaces should also provide an appropriate reference for measurements on surfaces passivated by more complex overlayers, e.g. SAMs. In addition to providing 
model passivated InAs surfaces, the sulfur passivation treatment also satisfies a variety of criteria desirable for preparation of surfaces for sensor applications.

\section{Acknowledgements}

We would like to thank Dr. Paul Sheehan for his help with the AFM measurements and Dr. Lane Baker for useful discussions of surface chemistry in organic solutions. This work was supported by the Office of Naval Research.

\section{References}

[1] V.N. Bessolov, M.V. Lebedev, Semiconductors 32 (1998) 1141.

[2] C.A. Mead, W.G. Spitzer, Phys. Rev. A 134 (1964) A713.

[3] M.V. Lebedev, Progr. Surf. Sci. 70 (2002) 153.

[4] F. Seker, K. Meeker, T.F. Kuech, A.B. Ellis, Chem. Rev. 100 (2000) 2505.

[5] W.E. Spicer, N. Newman, C.J. Spindt, Z. Lilientalweber, E.R. Weber, J. Vac. Sci. Technol. A 8 (1990) 2084.

[6] A.M. Green, W.E. Spicer, J. Vac. Sci. Technol. A 11 (1993) 1061.

[7] K. Gartsman, D. Cahen, A. Kadyshevitch, J. Libman, T. Moav, R. Naaman, A. Shanzer, V. Umansky, A. Vilan, Chem. Phys. Lett. 283 (1998) 301.

[8] K. Kajiyama, Y. Mizushim, S. Sakata, Appl. Phys. Lett. 23 (1973) 458.

[9] D.C. Tsui, Phys. Rev. Lett. 24 (1970) 303.

[10] J.W.G. Wildoer, C.J.P.M. Harmans, H. van Kempen, Phys. Rev. B 55 (1997) 16013.

[11] K. Kanisawa, M.J. Butcher, H. Yamaguchi, Y. Hirayama, Phys. Rev. Lett. 86 (2001) 3384.

[12] S. Bhargava, H.R. Blank, V. Narayanamurti, H. Kroemer, Appl. Phys. Lett. 70 (1997) 759.

[13] G.R. Bell, T.S. Jones, C.F. McConville, Appl. Phys. Lett. 71 (1997) 3688.

[14] P. De Padova, C. Quaresima, P. Perfetti, et al., Surf. Sci. 482 (2001) 587.

[15] M. Noguchi, K. Hirakawa, T. Ikoma, Phys. Rev. Lett. 66 (1991) 2243.

[16] L.O. Olsson, C.B.M. Andersson, M.C. Hakansson, J. Kanski, L. Ilver, U.O. Karlsson, Phys. Rev. Lett. 76 (1996) 3626.

[17] K. Smit, L. Koenders, W. Monch, J. Vac. Sci. Technol. B 7 (1989) 888.

[18] W. Monch, J. Vac. Sci. Technol. B 4 (1986) 1085.

[19] W. Monch, Europhys. Lett. 7 (1988) 275.

[20] J.F. Fan, H. Oigawa, Y. Nannichi, Jpn. J. Appl. Phys. 27 (1988) L1331.
[21] H.H. Wieder, J. Vac. Sci. Technol. 15 (1978) 1498

[22] M.S. Carpenter, M.R. Melloch, M.S. Lundstrom, S.P. Tobin, Appl. Phys. Lett. 52 (1988) 2157.

[23] Y. Nannichi, J.F. Fan, H. Oigawa, A. Koma, Jpn. J. Appl. Phys. 27 (1988) L2367.

[24] H. Oigawa, J.F. Fan, Y. Nannichi, H. Sugahara, M. Oshima, Jpn. J. Appl. Phys. 30 (1991) L322.

[25] Y.A. Kudryavtsev, E.B. Novikov, N.M. Stus, E.I. Chaikina, Semiconductors 26 (1992) 975.

[26] S. Ichikawa, N. Sanada, S. Mochizuki, Y. Esaki, Y. Fukuda, M. Shimomura, T. Abukawa, S. Kono, Phys. Rev. B 61 (2000) 12982.

[27] S. Ichikawa, N. Sanada, N. Utsumi, Y. Fukuda, J. Appl. Phys. 84 (1998) 3658.

[28] D.M. Poirier, J.H. Weaver, Surf. Sci. Spectra 2 (1993) 224.

[29] Y. Fukuda, Y. Suzuki, N. Sanada, M. Shimomura, S. Masuda, Phys. Rev. B 56 (1997) 1084.

[30] M. Katayama, M. Aono, H. Oigawa, Y. Nannichi, H. Sugahara, M. Oshima, Jpn. J. Appl. Phys. 30 (1991) L786.

[31] J. Tersoff, Phys. Rev. B 30 (1984) 4874.

[32] J. van Laar, A. Huijser, T.L. van Rooy, J. Vac. Sci. Technol. 14 (1977) 894.

[33] R.E. Allen, O.F. Sankey, J.D. Dow, Surf. Sci. 168 (1986) 376.

[34] D.Y. Petrovykh, M.J. Yang, J.P. Long, L.J. Whitman, in preparation.

[35] Y. Watanabe, F. Maeda, Appl. Surf. Sci. 117 (1997) 735.

[36] G. Eftekhari, Phys. Stat. Sol. A 161 (1997) 571.

[37] X.Y. Gong, T. Yamaguchi, H. Kan, T. Makino, K. Ohshimo, M. Aoyama, M. Kumagawa, N.L. Rowell, R. Rinfret, Appl. Surf. Sci. 114 (1997) 388.

[38] J.W. Kim, M.G. Kang, H.H. Park, Thin Solid Films 356 (1999) 423.

[39] H. Peisert, P. Streubel, T. Chasse, R. Szargan, Fresenius J. Anal. Chem. 358 (1997) 201.

[40] G. Hollinger, R. Skheytakabbani, M. Gendry, Phys. Rev. B 49 (1994) 11159.

[41] T. Yamaguchi, K. Ohshimo, A.H. Jayatissa, M. Aoyama, X.Y. Gong, T. Makino, H. Kan, Proc. SPIE 2873 (1996) 282.

[42] The Merck Index, Merck, Rahway NJ, 1989.

[43] W.F. Linke, Solubilities, ACS, Washington DC, 1965.

[44] V.N. Bessolov, E.V. Konenkova, M.V. Lebedev, Mater. Sci. Eng. B 44 (1997) 376.

[45] V.N. Bessolov, Y.V. Zhilyaev, E.V. Konenkova, M.V. Lebedev, Tech. Phys. 43 (1998) 983.

[46] Y. Cui, Q.Q. Wei, H.K. Park, C.M. Lieber, Science 293 (2001) 1289.

[47] X.F. Duan, C.M. Lieber, Adv. Mater. 12 (2000) 298.

[48] H. Yamaguchi, J.L. Sudijono, B.A. Joyce, T.S. Jones, C. Gatzke, R.A. Stradling, Phys. Rev. B 58 (1998) R4219.

[49] H. Yamaguchi, R. Dreyfus, Y. Hirayama, S. Miyashita, Appl. Phys. Lett. 78 (2001) 2372.

[50] Z.Q. He, L. Ilver, J. Kanski, P.O. Nilsson, P. Songsiriritthigul, G. Holmen, U.O. Karlsson, Appl. Surf. Sci. 104 (1996) 608 . 
[51] R.J. Haug, H. Munekata, L.L. Chang, Surf. Sci. 263 (1992) 374.

[52] K.A. Cheng, C.H. Yang, M.J. Yang, J. Appl. Phys. 88 (2000) 5272.

[53] W.J. Royea, A. Juang, N.S. Lewis, Appl. Phys. Lett. 77 (2000) 1988.
[54] A. Bansal, N.S. Lewis, J. Phys. Chem. B 102 (1998) 1067.

[55] T.A. Tanzer, P.W. Bohn, I.V. Roshchin, L.H. Greene, J.F. Klem, Appl. Phys. Lett. 75 (1999) 2794.

[56] T. Baum, S. Ye, K. Uosaki, Langmuir 15 (1999) 8577. 